\title{
Biology of HIV Infection
}

National Cancer Institute

\section{Source}

National Cancer Institute. Biology of HIV Infection. NCI Thesaurus. Code C18650.

Delineate the molecular and cellular mechanisms of establishment and systemic spread of infection and their impact on disease progression. 